\title{
ZALCMAN CONJECTURE AND HANKEL DETERMINANT OF ORDER THREE FOR STARLIKE AND CONVEX FUNCTIONS ASSOCIATED WITH SHELL-LIKE CURVES
}

\begin{abstract}
The aim of this article is to estimate an upper bound of $\left|H_{3}(1)\right|$, the Zalcman coefficient functional for $n=3$ and $n=4$, and also to investigate the fifth, sixth, seventh coefficients of starlike and convex functions associated with shell-like curves. Similar type of outcomes are estimated for the functions $f^{-1}$ and $\frac{z}{f(z)}$.

Key words: Analytic function, Function with positive real part, Starlike function, Subordination, Zalcman conjecture, Shell-like curve, Hankel determinant
\end{abstract}

2010 Mathematical Subject Classification: Primary 30C45;

Secondary 30C50, 30C80

1. Introduction. Denote by $\mathcal{A}$ the class of all normalized holomorphic functions $f$ of the form

$$
f(z)=z+\sum_{n \geq 2} a_{n} z^{n} \quad \forall z \in \mathcal{U}
$$

in the unit $\operatorname{disc} \mathcal{U}=\{z \in \mathbb{C}:|z|<1\}$. Let $\mathcal{S}$ be a subclass of $\mathcal{A}$ consisting of univalent functions in $\mathcal{U}$. A function $f \in S$ is a starlike function iff

$$
\mathcal{R} e\left(z f^{\prime}(z) / f(z)\right)>0 ; \forall z \in \mathcal{U} \text {. }
$$

A function $f \in \mathcal{S}$ is a convex function iff

$$
\mathcal{R} e\left(1+\left(z f^{\prime \prime}(z) / f^{\prime}(z)\right)\right)>0 ; \forall z \in \mathcal{U} .
$$

Let $\mathcal{P}$ be the family of holomorphic functions $p$ in $\mathcal{U}$ with the conditions $\mathcal{R} e\{p(z)\}>0$ and $p(0)=1$ represented in the form

$$
p(z)=1+c_{1} z+c_{2} z^{2}+c_{3} z^{3}+\ldots
$$

(C) Petrozavodsk State University, 2020 
For $f \in \mathcal{S}$, Lawrence Zalcman conjectured that $\left|a_{n}^{2}-a_{2 n-1}\right| \leq(n-1)^{2}$. This conjecture was proved by Krushkal [10] for $n=3,4,5,6$. It was also considered by Ma [11] and Ravichandran [14]. Equality holds for the Koebe function and its rotations. A holomorphic function $F$ is subordinate to another holomorphic function $h$, denoted by $F \prec h$, iff there exists a Schwarz function $w$ in $\mathcal{U}$ with the conditions $w(0)=0$, and $|w(z)|-1<0$, such that $F(z)=h(w(z))$. The Hadamard product of two holomorphic functions $f(z)=z+\sum_{n=2}^{\infty} a_{n} z^{n}$ and $g(z)=z+\sum_{n=2}^{\infty} g_{n} z^{n}$ in $\mathcal{A}$, is defined as

$$
(f \star g)(z)=z+\sum_{n=2}^{\infty} a_{n} g_{n} z^{n} .
$$

In the year 1966, Pommerenke [12] has denoted the Hankel determinant by $H_{q}(n)$ and defined it as

$$
H_{q}(n)=\left|\begin{array}{ccccc}
a_{n} & a_{n+1} & a_{n+2} & \ldots \ldots & a_{n+q-1} \\
a_{n+1} & a_{n+2} & a_{n+3} & \ldots \ldots & a_{n+q} \\
\ldots & \ldots & \ldots & \ldots \ldots & \ldots \\
\ldots & \ldots & \ldots & \ldots \ldots & \ldots \\
a_{n+q-1} & a_{n+q} & a_{n+q+1} & \ldots \ldots & a_{n+2 q-2}
\end{array}\right|
$$

Here $n, q \in \mathbb{N},\left\langle a_{n}\right\rangle$ is a sequence of real or complex numbers. For different values of $n$ and $q$, one obtains different Hankel determinants and also some particular cases of Fekete-Szegö coefficient functional. Many authors [1], [4], [6], [15], [16], [18] have studied the Fekete-Szegö coefficient functional for different subclasses of univalent, multivalent, and holomorphic functions. For $n=q=2$, the relation (3) reduces to $\left|H_{2}(2)\right|=\left|a_{2} a_{4}-a_{3}^{2}\right|$. This is known as the second-order Hankel determinant. Several authors [7], [8], [18] have studied this determinant for different subclasses of holomorphic functions. For $n=1$ and $q=3$, from the determinant $H_{q}(n)$ after applying the triangle inequality, one gets an upper bound for the third order Hankel determinant, given by

$$
\left|H_{3}(1)\right| \leq\left|a_{3}\right|\left|a_{2} a_{4}-a_{3}^{2}\right|+\left|a_{4}\right|\left|a_{4}-a_{2} a_{3}\right|+\left|a_{5}\right|\left|a_{3}-a_{2}^{2}\right|
$$

This is known as the third Hankel determinant for $a_{1}=1$. Babalola [3], Srivastava [17], Vamshee Krishna and Ram Reddy [18] have studied the third order Hankel determinant $H_{3}(1)$ for different subfamilies of analytic functions. 
Raina and Sokół [4], [13] have used the function $\mathbf{q}(z)$ as the superordinating function. The function $\mathbf{q}(z)=\sqrt{1+z^{2}}+z$ is analytic and univalent on $\mathbb{C} \backslash\{i,-i\}$, which maps the unit disc onto a shell shaped region on the right half plane. It is symmetric with respect to the real axis from 0.4 to 2.41. It is a function with positive real part with $\mathbf{q}(0)=\mathbf{q}^{\prime}(0)=1$.



Figure 1: Shell shaped region

Using $\mathbf{q}(z)$, they have defined $S^{*}(\mathbf{q})$ as shown below and studied the initial coefficients, Fekete-Szegö coefficient functional, Hankel determinant of order two for the function $f$ in $S^{*}(\mathbf{q})$.

Definition 1. $f \in \mathcal{A}$ is a function of the class $S^{*}(\mathbf{q})$ iff

$$
\frac{z f^{\prime}(z)}{f(z)} \prec \sqrt{1+z^{2}}+z .
$$

Definition 2. $f, g \in \mathcal{A}$ are two functions of the class $S_{g}^{*}(\mathbf{q})$ iff

$$
\frac{z\left((f * g)^{\prime}(z)\right)}{(f * g)(z)} \prec \sqrt{1+z^{2}}+z .
$$

Definition 3. $f \in \mathcal{A}$ is a function of the $\operatorname{class} \mathcal{C}(\mathbf{q})$ iff

$$
\left(1+\frac{z f^{\prime \prime}(z)}{f^{\prime}(z)}\right) \prec \sqrt{1+z^{2}}+z .
$$


Subordination results of this kind for various subclasses of analytic functions were obtained by several mathematicians, e.g., [2], [5], [17]. Recently, Srivastava revived the study of Hankel determinants, his pioneering work on the subject was followed by a huge flood of papers dealing with coefficient inequalities, Hankel determinants of order two and three of univalent and holomorphic functions. Different superordinating functions and their geometrical interpretations have motivated further research of the subject of geometric function theory. Functions like $\phi(z)=\frac{1+z}{1-z}, \frac{1+A z}{1+B z}$, $\sqrt{1+z}, e^{z}, \sin z$, the Fibonacci sequence are some among them to quote.

Our work was motivated by Babalola [3], Sharma [15], Vamshee Krishna [18], Ravichandran et al. [14], Srivastava et al. [16], [17] in general, and Sokół [4], [13] in particular, In this paper, we evaluate the bounds on $a_{5}, a_{6}, a_{7}$, and $H_{3}(1)$ for $f \in S^{*}(\mathbf{q})$. For $f \in S^{*}(\mathbf{q})$, we estimate the bounds for Zalcman's functional for $n=3,4$. Also, we define the class $\mathcal{C}(\mathbf{q})$ and make a similar study associated with shell-like curves for a function $f \in \mathcal{C}(\mathbf{q})$.

\section{Preliminaries.}

Lemma 1. [12] If $p \in \mathcal{P}$ is of the form $p(z)=1+c_{1} z+c_{2} z^{2}+\ldots$, then $\left|c_{n}\right| \leq 2 \quad \forall n \in \mathbb{N}$.

Lemma 2. [9] For a Schwarz function $w(z)=c_{1} z+c_{2} z^{2}+\ldots$, and for any $\mu \in \mathbb{C}$ we have

$$
\left|c_{2}-\mu c_{1}^{2}\right| \leq \max \{1,|\mu|\} .
$$

\section{Coefficient estimates for $f \in S^{*}(\mathbf{q})$.}

Theorem 1. If $f \in S^{*}(\mathbf{q})$ is of the form $f(z)=z+a_{1} z+a_{2} z^{2}+\ldots$, then

$$
\left|a_{5}\right| \leq 13 / 24, \quad\left|a_{6}\right| \leq 29 / 30, \quad\left|a_{7}\right| \leq 309 / 288 .
$$

Proof. As $f \in S^{*}(\mathbf{q})$, by using subordination we get

$$
\begin{aligned}
\frac{z f^{\prime}(z)}{f(z)} & =w(z)+\sqrt{1+w^{2}(z)} . \\
z f^{\prime}(z)-f(z) w(z) & =f(z) \sqrt{1+w^{2}(z)} .
\end{aligned}
$$

Here $w$ is the Schwarz function with the conditions $w(0)=0$ and $|w(z)|<1$ for $|z|<1$, which can be represented as

$$
w(z)=\sum_{n \geq 1} c_{n} z^{n}, \quad \forall n \in \mathbb{N} \text { with }\left|c_{n}\right| \leq 1 .
$$


From relations (6) and (7) we have

$$
\begin{gathered}
\sqrt{1+w^{2}(z)}=1+\frac{c_{1}^{2}}{2} z^{2}+\left(c_{1} c_{2}\right) z^{3}+\left(c_{1} c_{3}+\frac{c_{2}^{2}}{2}-\frac{c_{1}^{4}}{8}\right) z^{4}+ \\
+\left(c_{1} c_{4}+c_{2} c_{3}-\frac{c_{1}^{3} c_{2}}{2}\right) z^{5}+\left(\frac{c_{3}^{2}}{2}+c_{1} c_{5}+c_{2} c_{4}-\frac{3 c_{1}^{2} c_{2}^{2}}{4}-\frac{c_{1}^{3} c_{3}}{2}+\frac{c_{1}^{6}}{16}\right) z^{6}+\ldots
\end{gathered}
$$

and

$$
\begin{aligned}
f(z) & \sqrt{1+w^{2}(z)}=z+a_{2} z^{2}+\left(\frac{c_{1}^{2}}{2}+a_{3}\right) z^{3}+\left(c_{1} c_{2}+\frac{a_{2} c_{1}^{2}}{2}+a_{4}\right) z^{4}+ \\
& +\left(c_{1} c_{3}+\frac{c_{2}^{2}}{2}-\frac{c_{1}^{4}}{8}+a_{2} c_{1} c_{2}+\frac{a_{3} c_{1}^{2}}{2}+a_{5}\right) z^{5}+\left(c_{1} c_{4}+c_{2} c_{3}-\right. \\
& \left.-\frac{c_{1}^{3} c_{2}}{2}+\frac{a_{2} c_{2}^{2}}{2}+a_{2} c_{1} c_{3}-\frac{a_{2} c_{1}^{4}}{8}+a_{3} c_{1} c_{2}+\frac{a_{4} c_{1}^{2}}{2}+a_{6}\right) z^{6}+ \\
& +\left(\frac{c_{3}^{2}}{2}-\frac{3 c_{1}^{2} c_{2}^{2}}{4}-\frac{c_{1}^{3} c_{3}}{2}+\frac{c_{1}^{6}}{16}-\frac{a_{2} c_{1}^{3} c_{2}}{2}+\frac{a_{5} c_{1}^{2}}{2}+\frac{a_{3} c_{2}^{2}}{2}-\frac{a_{3} c_{1}^{4}}{8}+\right. \\
& \left.+c_{1} c_{5}+c_{2} c_{4}+a_{2} c_{1} c_{4}+a_{2} c_{2} c_{3}+a_{4} c_{1} c_{2}+a_{3} c_{1} c_{3}+a_{7}\right) z^{7}+\ldots
\end{aligned}
$$

Further,

$$
\begin{aligned}
& z f^{\prime}(z)-w(z) f(z)=z+\left(2 a_{2}-c_{1}\right) z^{2}+\left(3 a_{3}-a_{2} c_{1}-c_{2}\right) z^{3}+ \\
& \quad+\left(4 a_{4}-a_{3} c_{1}-a_{2} c_{2}-c_{3}\right) z^{4}+\left(5 a_{5}-a_{4} c_{1}-a_{3} c_{2}-a_{2} c_{3}-c_{4}\right) z^{5}+ \\
& \quad+\left(6 a_{6}-a_{5} c_{1}-a_{4} c_{2}-a_{3} c_{3}-a_{2} c_{4}-c_{5}\right) z^{6}+ \\
& \quad+\left(7 a_{7}-a_{6} c_{1}-a_{5} c_{2}-a_{4} c_{3}-a_{3} c_{4}-a_{2} c_{5}-c_{6}\right) z^{7}+\ldots
\end{aligned}
$$

From (8) and (9), upon equating the coefficients of the same powers of $z$,

$$
\begin{gathered}
a_{2}=c_{1}, \\
a_{3}=\frac{1}{2}\left(c_{2}+\frac{3}{2} c_{1}^{2}\right), \\
a_{4}=\frac{1}{3}\left(\frac{5}{4} c_{1}^{3}+\frac{5}{2} c_{1} c_{2}+c_{3}\right), \\
a_{5}=\frac{1}{4}\left(\frac{7}{3} c_{1} c_{3}+\frac{17}{6} c_{1}^{2} c_{2}+\frac{2}{3} c_{1}^{4}+c_{2}^{2}+c_{4}\right), \\
a_{6}=\frac{1}{60}\left(27 c_{1} c_{4}+20 c_{2} c_{3}+19 c_{1}^{3} c_{2}+20 c_{1} c_{2}^{2}+30 c_{1}^{2} c_{3}+3 c_{1}^{5}+12 c_{5}\right), \\
a_{7}=\frac{1}{2880}\left(400 c_{3}^{2}+1056 c_{1} c_{5}+840 c_{2} c_{4}+780 c_{1}^{2} c_{2}^{2}+700 c_{1}^{3} c_{3}+\right.
\end{gathered}
$$




$$
\left.+49 c_{1}^{6}+1116 c_{1}^{2} c_{4}+1720 c_{1} c_{2} c_{3}+332 c_{1}^{4} c_{2}+240 c_{2}^{3}+480 c_{6}\right) .
$$

Raina and Sokół [13] estimated the bounds of the second, third, and fourth coefficients as $\left|a_{2}\right| \leq 1,\left|a_{3}\right| \leq 3 / 4$ and $\left|a_{4}\right| \leq 1 / 2$. To estimate the bounds of the fifth, sixth, and seventh coefficients, we establish some properties of $c_{n}$ involved in (7). The function $p(z)$ is given by

$$
\frac{1+w(z)}{1-w(z)}=1+p_{1} z+p_{2} z^{2}+\ldots=p(z) .
$$

The Caratheodory function is defined by the property $\mathcal{R} e\{p(z)\}>0$ in $\mathcal{U}$, whose coefficients satisfy the condition

$$
\left|p_{k}\right| \leq 2 \quad \forall k \in \mathbb{N}
$$

Equating the coefficients of the same powers of $z$ in relation (16), we get

$$
\begin{gathered}
p_{1}=2 c_{1}, \\
p_{2}=2\left(c_{1}^{2}+c_{2}\right), \\
p_{3}=2\left(c_{1}^{3}+2 c_{1} c_{2}+c_{3}\right), \\
p_{4}=2\left(c_{1}^{4}+3 c_{1}^{2} c_{2}+2 c_{1} c_{3}+c_{2}^{2}+c_{4}\right), \\
p_{6}=2\left(c_{1}^{6}+5 c_{1}^{4} c_{2}+4 c_{1}^{2} c_{2}^{2}+4 c_{1}^{3} c_{3}+c_{2}^{3}+c_{3}^{2}+3 c_{1} c_{2} c_{3}+3 c_{1}^{2} c_{4}+2 c_{2} c_{4}+2 c_{1} c_{5}+c_{6}\right) \\
\text { and } \quad \\
p_{7}=2\left(c_{1}^{7}+3 c_{1} c_{3}^{2}+6 c_{1}^{5} c_{2}+6 c_{1}^{3} c_{2}^{2}+4 c_{1} c_{2}^{3}+5 c_{1}^{4} c_{3}+9 c_{1}^{2} c_{2} c_{3}+3 c_{2}^{2} c_{3}+\right. \\
\left.+4 c_{1}^{3} c_{4}+3 c_{1} c_{2} c_{4}+2 c_{3} c_{4}+3 c_{1}^{2} c_{5}+2 c_{2} c_{5}+2 c_{1} c_{6}+c_{7}\right) .
\end{gathered}
$$

Apply the condition in (17) to relations (18) to (24) and get

$$
\begin{gathered}
\left|c_{1}\right| \leq 1, \\
\left|c_{1}^{2}+c_{2}\right| \leq 1, \\
\left|c_{1}^{3}+2 c_{1} c_{2}+c_{3}\right| \leq 1, \\
\left|c_{1}^{4}+3 c_{1}^{2} c_{2}+2 c_{1} c_{3}+c_{2}^{2}+c_{4}\right| \leq 1, \\
\left|c_{1}^{5}+3 c_{1} c_{2}^{2}+3 c_{1}^{2} c_{3}+4 c_{1}^{3} c_{2}+2 c_{2} c_{3}+2 c_{1} c_{4}+c_{5}\right| \leq 1,
\end{gathered}
$$




$$
\begin{gathered}
\mid c_{1}^{6}+5 c_{1}^{4} c_{2}+4 c_{1}^{2} c_{2}^{2}+4 c_{1}^{3} c_{3}+c_{2}^{3}+c_{3}^{2}+3 c_{1} c_{2} c_{3}+3 c_{1}^{2} c_{4}+ \\
+2 c_{2} c_{4}+2 c_{1} c_{5}+c_{6} \mid \leq 1
\end{gathered}
$$

and

$$
\begin{gathered}
\mid c_{1}^{7}+3 c_{1} c_{3}^{2}+6 c_{1}^{5} c_{2}+6 c_{1}^{3} c_{2}^{2}+4 c_{1} c_{2}^{3}+5 c_{1}^{4} c_{3}+9 c_{1}^{2} c_{2} c_{3}+3 c_{2}^{2} c_{3}+ \\
+4 c_{1}^{3} c_{4}+3 c_{1} c_{2} c_{4}+2 c_{3} c_{4}+3 c_{1}^{2} c_{5}+2 c_{2} c_{5}+2 c_{1} c_{6}+c_{7} \mid \leq 1
\end{gathered}
$$

From the relation (13), we have

$$
\begin{gathered}
a_{5}=\frac{1}{24}\left\{14 c_{1} c_{3}+6 c_{2}^{2}+4 c_{1}^{4}+17 c_{1}^{2} c_{2}+6 c_{4}\right\} \\
a_{5}=\frac{7}{24}\left(c_{1}^{4}+3 c_{1}^{2} c_{2}+2 c_{1} c_{3}+c_{2}^{2}+c_{4}\right)- \\
-\frac{3}{24}\left(c_{1}^{2}+c_{2}\right)^{2}+\frac{1}{12} c_{2}\left(c_{1}^{2}+c_{2}\right)-\frac{c_{4}}{24} .
\end{gathered}
$$

By applying the triangle inequality to relation (32), we get

$$
\begin{aligned}
\left|a_{5}\right| \leq \frac{7}{24}\left|c_{1}^{4}+3 c_{1}^{2} c_{2}+2 c_{1} c_{3}+c_{2}^{2}+c_{4}\right|+ & \frac{3}{24}\left|\left(c_{1}^{2}+c_{2}\right)^{2}\right|+ \\
& +\frac{1}{12}\left|c_{2}\left(c_{1}^{2}+c_{2}\right)\right|+\left|\frac{c_{4}}{24}\right| .
\end{aligned}
$$

We know that coefficients of the function $w(z)$ satisfy $\left|c_{n}\right| \leq 1$. By applying relations (26) and (28) to relation (33), we get

$$
\left|a_{5}\right| \leq \frac{7}{24}+\frac{3}{24}+\frac{2}{24}+\frac{1}{24}=\frac{13}{24} .
$$

Using relation (14), we can estimate the bound on the sixth coefficient.

$$
\begin{aligned}
a_{6}=\frac{1}{60} & {\left[3\left(c_{1}^{5}+2 c_{1} c_{4}+3 c_{1}^{2} c_{3}+4 c_{1}^{3} c_{2}+3 c_{1} c_{2}^{2}+2 c_{2} c_{3}+c_{5}\right)+\right.} \\
& +7 c_{1}\left(c_{1}^{4}+3 c_{1}^{2} c_{2}+2 c_{1} c_{3}+c_{2}^{2}+c_{4}\right)-7 c_{1}^{2}\left(c_{1}^{3}+2 c_{1} c_{2}+c_{3}\right)+ \\
& \left.+14 c_{3}\left(c_{1}^{2}+c_{2}\right)+4 c_{1} c_{2}^{2}+14 c_{1} c_{4}+9 c_{5}\right] .
\end{aligned}
$$

Using the triangle inequality to relation (35), we get

$$
\left|a_{6}\right| \leq \frac{1}{60}\left[3\left|c_{1}^{5}+2 c_{1} c_{4}+3 c_{1}^{2} c_{3}+4 c_{1}^{3} c_{2}+3 c_{1} c_{2}^{2}+2 c_{2} c_{3}+c_{5}\right|+\right.
$$




$$
\begin{aligned}
& +7\left|c_{1}\right|\left|c_{1}^{4}+3 c_{1}^{2} c_{2}+2 c_{1} c_{3}+c_{2}^{2}+c_{4}\right|+7\left|c_{1}\right|^{2}\left|c_{1}^{3}+2 c_{1} c_{2}+c_{3}\right|+ \\
& \left.+14\left|c_{3}\right|\left|c_{1}^{2}+c_{2}\right|+4\left|c_{1}\right|\left|c_{2}\right|^{2}+14\left|c_{1}\right|\left|c_{4}\right|+9\left|c_{5}\right|\right]
\end{aligned}
$$

We know that coefficients of the function $w(z)$ satisfy $\left|c_{n}\right| \leq 1$. By applying relations (26) to (29) to relation (36), it is reduced to

$$
\left|a_{6}\right| \leq \frac{29}{30}
$$

Using relation (15), we can estimate the bound on the seventh coefficient

$$
\begin{aligned}
a_{7} & =\frac{1}{2880}\left[2 0 0 \left(c_{1}^{6}+5 c_{1}^{4} c_{2}+4 c_{1}^{2} c_{2}^{2}+4 c_{1}^{3} c_{3}+c_{2}^{3}+c_{3}^{2}+3 c_{1} c_{2} c_{3}+3 c_{1}^{2} c_{4}+\right.\right. \\
& \left.+2 c_{2} c_{4}+2 c_{1} c_{5}+c_{6}\right)+50 c_{1}\left(c_{1}^{5}+3 c_{1} c_{2}^{2}+3 c_{1}^{2} c_{3}+4 c_{1}^{3} c_{2}+2 c_{2} c_{3}+\right. \\
& \left.+2 c_{1} c_{4}+c_{5}\right)+40 c_{2}\left(c_{1}^{4}+3 c_{1}^{2} c_{2}+2 c_{1} c_{3}+c_{2}^{2}+c_{4}\right)-200 c_{1}^{2}\left(c_{1}^{4}+3 c_{1}^{2} c_{2}+\right. \\
& \left.+2 c_{1} c_{3}+c_{2}^{2}+c_{4}\right)+200 c_{3}\left(c_{1}^{3}+2 c_{1} c_{2}+c_{3}\right)-50 c_{1}^{3}\left(c_{1}^{3}+2 c_{1} c_{2}+c_{3}\right)+ \\
& +400 c_{4}\left(c_{1}^{2}+c_{2}\right)+540 c_{1} c_{2} c_{3}-90 c_{1}^{2} c_{2}\left(c_{1}^{2}+c_{2}\right)+50 c_{1}^{4}\left(c_{1}^{2}+c_{2}\right)- \\
& \left.-168 c_{1}^{4} c_{2}+216 c_{1}^{2} c_{4}+280 c_{6}+606 c_{1} c_{5}\right] .
\end{aligned}
$$

By applying the triangle inequality to relation (37) and using relations (26) to (30), we get

$$
\left|a_{7}\right| \leq \frac{309}{288}
$$

\section{The coefficient functional for $f \in S^{*}(\mathbf{q})$}

Theorem 2. If $f \in S^{*}(\mathbf{q})$ is of the form $f(z)=z+a_{1} z+a_{2} z^{2}+\cdots$, then

$$
\left|a_{2} a_{3}-a_{4}\right| \leq 1
$$

Proof. If $f \in S^{*}(\mathbf{q})$ then, from Theorem 1, upon using the values of $a_{2}, a_{3}, a_{4}$ from Equations (10), (11), and (12), obtain

$$
a_{2} a_{3}-a_{4}=\frac{c_{1}^{3}}{3}+\frac{c_{1}}{3}\left(c_{1}^{2}+c_{2}\right)-\frac{1}{3}\left(c_{1}^{3}+2 c_{1} c_{2}+c_{3}\right) .
$$

By applying the triangle inequality,

$$
\left|a_{2} a_{3}-a_{4}\right| \leq\left|\frac{c_{1}^{3}}{3}\right|+\left|\frac{c_{1}}{3}\left(c_{1}^{2}+c_{2}\right)\right|+\left|\frac{1}{3}\left(c_{1}^{3}+2 c_{1} c_{2}+c_{3}\right)\right| .
$$


From relations (25), (26), and (27), obtain

$$
\left|a_{2} a_{3}-a_{4}\right| \leq \frac{1}{3}+\frac{1}{3}+\frac{1}{3}=1 .
$$

\section{The third Hankel Determinant for $f \in S^{*}(\mathbf{q})$.}

Theorem 3. If $f \in S^{*}(\mathbf{q})$ is of the form $f(z)=z+a_{1} z+a_{2} z^{2}+\cdots$, then

$$
\left|H_{3}(1)\right| \leq \frac{265}{192}
$$

Proof. Due to Raina and Sokół [13], $\left|a_{2}\right| \leq 1,\left|a_{3}\right| \leq 3 / 4,\left|a_{4}\right| \leq 1 / 2, \mid a_{2} a_{4}-$ $a_{3}^{2} \mid \leq 39 / 48$ and $\left|a_{3}-a_{2}^{2}\right| \leq 1 / 2$. Substituting these bounds, relations (34) and (38) in the relation (4), we get

$$
\begin{gathered}
\left|H_{3}(1)\right| \leq \frac{3}{4}\left(\frac{39}{48}\right)+\frac{1}{2}(1)+\frac{13}{24}\left(\frac{1}{2}\right) . \\
\left|H_{3}(1)\right| \leq \frac{265}{192} .
\end{gathered}
$$

\section{Fekete-Szegö inequality for different functions.}

Theorem 4. Let $f^{-1}(z)=z+\sum_{n=2}^{\infty} d_{n} z^{n}$ be the inverse function of $f$. For any $\mu \in \mathbb{C}$ and $f \in S^{*}(\mathbf{q})$ of the form $f(z)=z+a_{1} z+a_{2} z^{2}+\ldots$, we get

$$
\left|d_{3}-\mu d_{2}^{2}\right| \leq \frac{1}{2} \max \left\{1,\left|\frac{5-4 \mu}{4}\right|\right\} .
$$

Proof. By the definition of the inverse function, we have

$$
\begin{aligned}
& f\left(f^{-1}(z)\right)=f^{-1}(f(z))=z . \\
& \text { Let } f^{-1}(z)=z+\sum_{n=2}^{\infty} d_{n} z^{n} .
\end{aligned}
$$

From relations (1) and (40), it can be reduced to

$$
f^{-1}\left(z+a_{2} z^{2}+a_{3} z^{3}+\ldots\right)=z .
$$


From (40) and (41) one obtains

$$
z+z^{2}\left(a_{2}+d_{2}\right)+z^{3}\left(a_{3}+2 a_{2} d_{2}+d_{3}\right)+\cdots=z
$$

Comparing the coefficients of $z^{2}$ and $z^{3}$ on both sides, one can see that

$$
a_{2}+d_{2}=0
$$

and

$$
a_{3}+2 a_{2} d_{2}+d_{3}=0
$$

Now, from (12) and (42) we get

$$
d_{2}=-c_{1}
$$

From (12), (13) and (43) we get

$$
d_{3}=-\frac{c_{2}}{2}+\frac{5 c_{1}^{2}}{4}
$$

Now consider

$$
\begin{gathered}
d_{3}-\mu d_{2}^{2}=\frac{1}{2}\left\{c_{2}-c_{1}^{2}\left(\frac{5-4 \mu}{4}\right)\right\} . \\
d_{3}-\mu d_{2}^{2}=\frac{1}{2}\left\{c_{2}-v_{1} c_{1}^{2}\right\}, \text { where } v_{1}=\frac{5-4 \mu}{4} .
\end{gathered}
$$

Applying Lemma 2 to relation (44), one gets estimate (39). This estimate is sharp, the equality is attained on the following functions

$$
\left|d_{3}-\mu d_{2}^{2}\right|= \begin{cases}\frac{1}{2}, & \text { if } p(z)=\frac{1+z^{2}}{1-z^{2}} \\ \left|\frac{5-4 \mu}{8}\right|, & \text { if } p(z)=\frac{1+z}{1-z}\end{cases}
$$

Theorem 5. For a function $f \in S^{*}(\mathbf{q})$ of the form $f(z)=z+a_{1} z+$ $+a_{2} z^{2}+\ldots$, for any $\mu \in \mathbb{C}$, and for $G(z)=\frac{z}{f(z)}=1+d_{1} z+d_{2} z^{2}+\ldots$, we get

$$
\left|d_{2}-\mu d_{1}^{2}\right| \leq \frac{1}{2} \max \left\{1,\left|\frac{1-4 \mu}{4}\right|\right\} .
$$

Proof. As $f \in S^{*}(\mathbf{q})$ and

$$
G(z)=\frac{z}{f(z)}=1+\sum_{n=1}^{\infty} d_{n} z^{n}
$$


Simplifying, one obtains that

$$
\frac{z}{f(z)}=1-a_{2} z+\left(a_{2}^{2}-a_{3}\right) z^{2}+\ldots
$$

From (46) and (47), upon equating the coefficients of the same powers of $z$, we have

$$
d_{1}=-a_{2}
$$

and

$$
d_{2}=a_{2}^{2}-a_{3} .
$$

From (10) and (48), we get $d_{1}=-c_{1}$.

From (10), (11) and (49), we get $d_{2}=\frac{c_{1}^{2}}{4}-\frac{c_{2}}{2}$.

Assuming that $\mu$ is a complex parameter, take

$$
\begin{gathered}
d_{2}-\mu d_{1}^{2}=\frac{1}{2}\left\{c_{2}-c_{1}^{2}\left(\frac{1-4 \mu}{4}\right)\right\} . \\
d_{2}-\mu d_{1}^{2}=\frac{1}{2}\left\{c_{2}-v_{2} c_{1}^{2}\right\}, \text { where } v_{2}=\frac{1-4 \mu}{4} .
\end{gathered}
$$

Applying Lemma 2 to relation (50), one gets (45), with equalities

$$
\left|d_{2}-\mu d_{1}^{2}\right|= \begin{cases}\frac{1}{2}, & \text { if } p(z)=\frac{1+z^{2}}{1-z^{2}} \\ \left|\frac{1-4 \mu}{8}\right|, & \text { if } p(z)=\frac{1+z}{1-z}\end{cases}
$$

Theorem 6. If $f \in S^{*}(\mathbf{q})$ is of the form $f(z)=z+a_{1} z+a_{2} z^{2}+\ldots$, then

$$
\left|a_{3}-\mu a_{2}^{2}\right| \leq \frac{1}{2 g_{3}} \max \left\{1,\left|\frac{2 \mu g_{3}-3 g_{2}^{2}}{2 g_{2}^{2}}\right|\right\} .
$$

Proof. Let $f \in S^{*}(\mathbf{q})$; then there exists a Schwarz function $w$ such that

$$
\frac{z\left((f * g)^{\prime}(z)\right)}{(f * g)(z)}=w(z)+\sqrt{1+w^{2}(z)} .
$$

The left-hand side of (52) has the expansion 


$$
\begin{aligned}
\frac{z\left((f * g)^{\prime}(z)\right)}{(f * g)(z)}=1+a_{2} g_{2} z & +\left(2 a_{3} g_{3}-a_{2}^{2} g_{2}^{2}\right) z^{2}+ \\
+ & \left(3 a_{4} g_{4}-3 a_{2} a_{3} g_{2} g_{3}+a_{2}^{3} g_{2}^{3}\right) z^{3}+\ldots
\end{aligned}
$$

Substituting the expansion $w(z)=c_{1} z+c_{2} z^{2}+\ldots$ into the right-hand side of (52), one obtains

$$
a_{2}=\frac{c_{1}}{g_{2}}, \quad a_{3}=\frac{c_{2}}{2 g_{3}}+\frac{3 c_{1}^{2}}{4 g_{3}}
$$

Consider

$$
\begin{gathered}
a_{3}-\mu a_{2}^{2}=\frac{1}{2 g_{3}}\left\{c_{2}-c_{1}^{2}\left(\frac{2 \mu g_{3}-3 g_{2}^{2}}{g_{2}^{2}}\right)\right\} \\
a_{3}-\mu a_{2}^{2}=\frac{1}{2 g_{3}}\left\{c_{2}-v_{3} c_{1}^{2}\right\}, \text { where } v_{3}=\frac{2 \mu g_{3}-3 g_{2}^{2}}{g_{2}^{2}} .
\end{gathered}
$$

Applying Lemma 2 to relation (54), one gets (51). The equality arises for

$$
\left|a_{3}-\mu a_{2}^{2}\right|= \begin{cases}\frac{1}{2 g_{3}}, & \text { if } p(z)=\frac{1+z^{2}}{1-z^{2}} \\ \left|\frac{2 \mu g_{3}-3 g_{2}^{2}}{4 g_{2}^{2} g_{3}}\right|, & \text { if } p(z)=\frac{1+z}{1-z} .\end{cases}
$$

7. Zalcman coefficient functional for $f \in S^{*}(\mathbf{q})$.

Theorem 7. If $f \in S^{*}(\mathbf{q})$ is of the form $f(z)=z+a_{1} z+a_{2} z^{2}+\ldots$, then

$$
\left|a_{3}^{2}-a_{5}\right| \leq \frac{53}{48}
$$

Proof. If $f \in S^{*}$ (q) then, from Theorem 1, upon using the values of $a_{3}$, $a_{5}$ from equations (11) and (13) one obtains that

$$
\begin{aligned}
a_{3}^{2}-a_{5}= & \frac{1}{4}\left(c_{2}+\frac{3}{2} c_{1}^{2}\right)^{2}-\frac{1}{4}\left(\frac{7}{3} c_{1} c_{3}+\frac{17}{6} c_{1}^{2} c_{2}+\frac{2}{3} c_{1}^{4}+c_{2}^{2}+c_{4}\right) . \\
a_{3}^{2}-a_{5}= & \frac{3}{8}\left(c_{1}^{2}+c_{2}\right)^{2}-\frac{7}{24}\left(c_{1}^{4}+3 c_{1}^{2} c_{2}+2 c_{1} c_{3}+c_{2}^{2}+c_{4}\right)+ \\
& +\frac{1}{4}\left(c_{1}^{2}\left(c_{1}^{2}+c_{2}\right)\right)-\frac{1}{12}\left(c_{2}\left(c_{1}^{2}+c_{2}\right)\right)+\frac{c_{1}^{4}}{16}-\frac{c_{4}}{24} .
\end{aligned}
$$


By applying the triangle inequality, we have

$$
\begin{aligned}
a_{3}^{2}-a_{5} \leq \mid \frac{3}{8} & \left(c_{1}^{2}+c_{2}\right)^{2}|+| \frac{7}{24}\left(c_{1}^{4}+3 c_{1}^{2} c_{2}+2 c_{1} c_{3}+c_{2}^{2}+c_{4}\right) \mid+ \\
& +\left|\frac{1}{4}\left(c_{1}^{2}\left(c_{1}^{2}+c_{2}\right)\right)\right|+\left|\frac{1}{12}\left(c_{2}\left(c_{1}^{2}+c_{2}\right)\right)\right|+\left|\frac{c_{1}^{4}}{16}\right|+\left|\frac{c_{4}}{24}\right| .
\end{aligned}
$$

From relations (7), (25), (26), and (28) we get

$$
\left|a_{3}^{2}-a_{5}\right| \leq \frac{3}{8}+\frac{7}{24}+\frac{1}{4}+\frac{1}{12}+\frac{1}{16}+\frac{1}{24}=\frac{53}{48} \text {. }
$$

Thus the result is proved for the case $n=3$ of the Zalcman conjecture for $f \in S^{*}(\mathbf{q})$.

Theorem 8. If $f \in S^{*}(\mathbf{q})$ is of the form $f(z)=z+a_{1} z+a_{2} z^{2}+\ldots$, then

$$
\left|a_{4}^{2}-a_{7}\right| \leq \frac{127}{96}
$$

Proof. If $f \in S^{*}(\mathbf{q})$ then, from Theorem 1, upon using the values of $a_{3}$, $a_{5}$ from equations (12) and (15) one obtains that

$$
\begin{aligned}
a_{4}^{2}-a_{7} & =\frac{1}{9}\left(\frac{5}{4} c_{1}^{3}+\frac{5}{2} c_{1} c_{2}+c_{3}\right)^{2}-\frac{1}{2880}\left(400 c_{3}^{2}+1056 c_{1} c_{5}+840 c_{2} c_{4}+\right. \\
& +780 c_{1}^{2} c_{2}^{2}+700 c_{1}^{3} c_{3}+49 c_{1}^{6}+1116 c_{1}^{2} c_{4}+1720 c_{1} c_{2} c_{3}+332 c_{1}^{4} c_{2}+ \\
& \left.+240 c_{2}^{3}+480 c_{6}\right) . \\
a_{4}^{2}-a_{7} & =\frac{25}{144}\left(c_{1}^{3}+2 c_{1} c_{2}+c_{3}\right)^{2}+\frac{1}{144} c_{3}^{2}-\frac{10}{144}\left(c_{1}^{3}+2 c_{1} c_{2}+c_{3}\right)\left(c_{3}\right)+ \\
& +\frac{1}{2880}\left[50 c_{1}\left(c_{1}^{5}+3 c_{1} c_{2}^{2}+3 c_{1}^{2} c_{3}+4 c_{1}^{3} c_{2}+2 c_{2} c_{3}+2 c_{1} c_{4}+c_{5}\right)+\right. \\
& +200\left(c_{1}^{6}+5 c_{1}^{4} c_{2}+4 c_{1}^{2} c_{2}^{2}+4 c_{1}^{3} c_{3}+c_{2}^{3}+c_{3}^{2}+3 c_{1} c_{2} c_{3}+3 c_{1}^{2} c_{4}+\right. \\
& \left.+2 c_{2} c_{4}+2 c_{1} c_{5}+c_{6}\right)+40 c_{2}\left(c_{1}^{4}+3 c_{1}^{2} c_{2}+2 c_{1} c_{3}+c_{2}^{2}+c_{4}\right)- \\
& -200 c_{1}^{2}\left(c_{1}^{4}+3 c_{1}^{2} c_{2}++2 c_{1} c_{3}+c_{2}^{2}+c_{4}\right)+200 c_{3}\left(c_{1}^{3}+2 c_{1} c_{2}+c_{3}\right)- \\
& -50 c_{1}^{3}\left(c_{1}^{3}+2 c_{1} c_{2}+c_{3}\right)+400 c_{4}\left(c_{1}^{2}+c_{2}\right)+540 c_{1} c_{2} c_{3}-90 c_{1}^{2} c_{2}\left(c_{1}^{2}+\right. \\
& \left.\left.+c_{2}\right)+50 c_{1}^{4}\left(c_{1}^{2}+c_{2}\right)-168 c_{1}^{4} c_{2}+216 c_{1}^{2} c_{4}+280 c_{6}+606 c_{1} c_{5}\right] .
\end{aligned}
$$


By using the triangle inequality and relations (7) and (25) - (31) to relation (55), we get the required result. Thus, the result is proved for the case $n=4$ of the Zalcman conjecture for $f \in S^{*}(\mathbf{q})$.

8. Coefficient estimates $f \in \mathcal{C}(\mathbf{q})$.

Theorem 9. If $f \in \mathcal{C}(\mathbf{q})$ is of the form $f(z)=z+a_{1} z+a_{2} z^{2}+\cdots$, then

$$
\left|a_{2}\right| \leq 1 / 2, \quad\left|a_{3}\right| \leq 1 / 4, \quad\left|a_{4}\right| \leq 7 / 24, \quad\left|a_{5}\right| \leq 3 / 40 .
$$

Proof. If the function $f \in \mathcal{C}(\mathbf{q})$, then from relation (5) we have

$$
f^{\prime}(z)+z f^{\prime \prime}(z)-f^{\prime}(z) w(z)=f^{\prime}(z) \sqrt{1+w^{2}(z)} .
$$

From the relations (7) and (56) we have

$$
\begin{gathered}
f^{\prime}(z) \sqrt{1+w^{2}(z)}=1+2 a_{2} z+\left(3 a_{3}+\frac{c_{1}^{2}}{2}\right) z^{2}+\left(4 a_{4}+a_{2} c_{1}^{2}+c_{1} c_{2}\right) z^{3}+ \\
+\left(5 a_{5}+2 a_{2} c_{1} c_{2}+c_{1} c_{3}+\frac{3 a_{3} c_{1}^{2}}{2}+\frac{c_{2}^{2}}{2}-\frac{c_{1}^{4}}{8}\right) z^{4}+\left(6 a_{6}+2 c_{1}^{2} a_{4}+\right. \\
\left.+c_{1} c_{4}+3 a_{3} c_{1} c_{2}+2 a_{2} c_{1} c_{3}+a_{2} c_{2}^{2}+c_{2} c_{3}-\frac{a_{2} c_{1}^{4}}{4}-\frac{c_{1}^{3} c_{2}}{2}\right) z^{5}+\ldots \quad(57)
\end{gathered}
$$

Further,

$$
\begin{array}{r}
f^{\prime}(z)+z f^{\prime \prime}(z)-f^{\prime}(z) w(z)=1+\left(4 a_{2}-c_{1}\right) z+\left(9 a_{3}-2 a_{2} c_{1}-c_{2}\right) z^{2}+ \\
+\left(16 a_{4}-3 a_{3} c_{1}-2 a_{2} c_{2}-c_{3}\right) z^{3}+\left(25 a_{5}-4 a_{4} c_{1}-3 a_{3} c_{2}-2 a_{2} c_{3}-c_{4}\right) z^{4}+ \\
+\left(36 a_{6}-5 a_{5} c_{1}-4 a_{4} c_{2}-3 a_{3} c_{3}-2 a_{2} c_{4}-c_{5}\right) z^{5}+\ldots
\end{array}
$$

From (57) and (58), upon comparing the coefficients of the same powers of $z$, we get

$$
\begin{gathered}
a_{2}=\frac{c_{1}}{2} \\
a_{3}=\frac{c_{1}^{2}}{4}+\frac{c_{2}}{6}=\frac{1}{6}\left(c_{2}+\frac{3 c_{1}^{2}}{2}\right) \\
a_{4}=\frac{5 c_{1}^{3}}{48}+\frac{5 c_{1} c_{2}}{24}-\frac{c_{3}}{12}=\frac{5}{48}\left(c_{1}^{3}+2 c_{1} c_{2}+c_{3}\right)-\frac{5 c_{3}}{48}-\frac{c_{3}}{12} \\
a_{5}=\frac{c_{1}^{4}}{30}+\frac{17 c_{1}^{2} c_{2}}{120}+\frac{c_{1} c_{3}}{12}+\frac{c_{2}^{2}}{20}+\frac{c_{4}}{20}, \\
a_{5}=\frac{1}{120}\left(5\left(c_{1}^{4}+3 c_{1}^{2} c_{2}+2 c_{1} c_{3}+c_{2}^{2}+c_{4}\right)+\left(c_{1}^{2}+c_{2}\right)^{2}-2 c_{1}^{4}+c_{4}\right) .
\end{gathered}
$$


By applying the triangle inequality to relations (59) - (62), also using relations (26)-(28), we get the required result.

Here the coefficients $a_{2}$ and $a_{3}$ have the best bounds for the function $f_{1}(z)$, which is defined as

$$
1+\left(z f_{1}^{\prime \prime}(z) / f_{1}^{\prime}(z)\right)=z+\left(1+z^{2}\right)^{\frac{1}{2}}, \quad f_{1}(z)=z+\sum_{n \geq 2} b_{n} z^{n}
$$

then $f_{1}^{\prime}(z)+z f_{1}^{\prime \prime}(z)-z f_{1}^{\prime}(z)=f_{1}^{\prime}(z) \sqrt{1+z^{2}}$.

After simplification, we get $b_{2}=1 / 2, \quad b_{3}=1 / 4$.

9. Coefficient functional for $f \in \mathcal{C}(\mathbf{q})$.

Theorem 10. If $f \in \mathcal{C}(\mathbf{q})$ is of the form $f(z)=z+a_{1} z+a_{2} z^{2}+\ldots$, then

$$
\left|a_{2} a_{3}-a_{4}\right| \leq 29 / 48
$$

Proof. If $f \in \mathcal{C}(\mathbf{q})$, then, from Theorem 9 and upon using the values of $a_{2}, a_{3}, a_{4}$ from equations (59), (60), and (61) one obtains

$$
a_{2} a_{3}-a_{4}=\frac{11 c_{1}^{3}}{48}-\frac{7 c_{1}}{24}\left(c_{1}^{2}+c_{2}\right)+\frac{1}{12}\left(c_{1}^{3}+2 c_{1} c_{2}+c_{3}\right) .
$$

By applying the triangle inequality, it is reduced to

$$
\left|a_{2} a_{3}-a_{4}\right| \leq\left|\frac{11 c_{1}^{3}}{48}\right|+\left|\frac{7 c_{1}}{24}\left(c_{1}^{2}+c_{2}\right)\right|+\left|\frac{1}{12}\left(c_{1}^{3}+2 c_{1} c_{2}+c_{3}\right)\right| .
$$

From relations (25), (26), and (27) we obtain that

$$
\left|a_{2} a_{3}-a_{4}\right| \leq \frac{11}{48}+\frac{7}{24}+\frac{1}{12}=\frac{29}{48} .
$$

\section{Fekete-Szegö inequality for $f \in \mathcal{C}(\mathbf{q})$.}

Theorem 11. If $f \in \mathcal{C}(\mathbf{q})$ is of the form $f(z)=z+a_{1} z+a_{2} z^{2}+\ldots$, then

$$
\left|a_{3}-\mu a_{2}^{2}\right| \leq \frac{1}{6} \max \left\{1,\left|\frac{3(\mu-1)}{2}\right|\right\}
$$

where $\mu$ is a complex number and the best bound is obtained.

Proof. If $f \in \mathcal{C}(\mathbf{q})$, then, from Theorem 9 and upon using the values of $a_{2}, a_{3}$, from equations (59) and (60) one obtains that

$$
a_{3}-\mu a_{2}^{2}=\frac{1}{6}\left(c_{2}-\nu_{1} c_{1}^{2}\right)
$$


where $\nu_{1}=\left(\frac{3(\mu-1)}{2}\right)$. By applying Lemma 2 to Equation (64), one obtains the result as in Equation (63). The sharpness is given below:

$$
\left|a_{3}-\mu a_{2}^{2}\right|= \begin{cases}1 / 6, & \text { if } p(z)=\frac{1+z^{2}}{1-z^{2}} \\ \left|\frac{\mu-1}{4}\right|, & \text { if } p(z)=\frac{1+z}{1-z}\end{cases}
$$

11. Second Order Hankel Determinant for $f \in \mathcal{C}(\mathbf{q})$.

Theorem 12. If $f \in \mathcal{C}(\mathbf{q})$ is of the form $f(z)=z+a_{1} z+a_{2} z^{2}+\ldots$, then

$$
\left|a_{2} a_{4}-a_{3}^{2}\right| \leq 31 / 144 \text {. }
$$

Proof. If $f \in \mathcal{C}(\mathbf{q})$, then from Theorem 9 and upon using the values of $a_{2}, a_{3}, a_{4}$, from equations (59), (60) and (61) one obtains that

$$
\left|a_{2} a_{4}-a_{3}^{2}\right|=\left|\frac{c_{1}\left(c_{1}^{3}+2 c_{1} c_{2}+c_{3}\right)}{96}+\frac{-12 c_{1}^{2} c_{2}+9 c_{1} c_{3}+8 c_{2}^{2}}{288}\right| .
$$

By applying the triangle inequality to relation (65) and also using relations (25) to (27), we get the required result.

12. An upper bound for $\left|H_{3}(1)\right|$ for $f \in \mathcal{C}(\mathbf{q})$.

Theorem 13. If $f \in \mathcal{C}(\mathbf{q})$ is of the form $f(z)=z+a_{1} z+a_{2} z^{2}+\ldots$, we have

$$
\left|H_{3}(1)\right| \leq \frac{1277}{5760}
$$

Proof. Substituting the results of Theorems 9, 10, 11, and 12 in the relation (4), we get

$$
\left|H_{3}(1)\right| \leq \frac{1}{4}\left(\frac{19}{144}\right)+\frac{7}{24}\left(\frac{29}{48}\right)+\frac{3}{40}\left(\frac{1}{6}\right)=\frac{1277}{5760} .
$$

13. Zalcman coefficient functional for $f \in \mathcal{C}(\mathbf{q})$.

Theorem 14. If $f \in \mathcal{C}(\mathbf{q})$ is of the form $f(z)=z+a_{1} z+a_{2} z^{2}+\cdots$, then

$$
\left|a_{3}^{2}-a_{5}\right| \leq \frac{7}{48}
$$


Proof. If $f \in \mathcal{C}(\mathbf{q})$, then, from Theorem 9 and upon using the values of $a_{3}, a_{5}$, from equations (60) and (62) one obtains that

$$
\begin{aligned}
& a_{3}^{2}-a_{5}=\frac{1}{720}\left(20\left(c_{1}^{2}+c_{2}\right)^{2}-30\left(c_{1}^{4}+3 c_{1}^{2} c_{2}+2 c_{1} c_{3}+c_{2}^{2}+c_{4}\right)+\right. \\
&\left.+20 c_{1}^{2}\left(c_{1}^{2}+c_{2}\right)-12 c_{2}\left(c_{1}^{2}+c_{2}\right)-6 c_{4}+11 c_{1}^{4}+6 c_{2}^{2}\right)
\end{aligned}
$$

By applying the triangle inequality to relation (66), we have

$$
\begin{aligned}
\left|a_{3}^{2}-a_{5}\right| & \leq \frac{1}{720}\left(20\left|c_{1}^{2}+c_{2}\right|^{2}+30\left|c_{1}^{4}+3 c_{1}^{2} c_{2}+2 c_{1} c_{3}+c_{2}^{2}+c_{4}\right|+\right. \\
& \left.+20\left|c_{1}\right|^{2}\left|c_{1}^{2}+c_{2}\right|+12\left|c_{2}\right|\left|c_{1}^{2}+c_{2}\right|+6\left|c_{4}\right|+11\left|c_{1}\right|^{4}+6\left|c_{2}\right|^{2}\right) .
\end{aligned}
$$

From relations (7), (25), (26), and (28), we get

$$
\left|a_{3}^{2}-a_{5}\right| \leq \frac{20}{720}+\frac{20}{720}+\frac{30}{720}+\frac{6}{720}+\frac{11}{720}+\frac{12}{720}+\frac{6}{720}=\frac{7}{48} .
$$

Acknowledgement. The authors are very much thankful to the Editor and the esteemed referees for their careful reading and valuable suggestions, which improved the paper.

The work presented in this paper is partially supported by DST/FIST grant no: SR/FST/MSI-101/2014, dated:14-01-2016.

\section{References}

[1] Ali R. M., Lee S. K., Ravichandran V., Supramaniam S. The Fekete-Szegö coefficient functional for transforms of analytic functions. Bull. Iran. Math. Soc., 2009, vol. 35, no. 2, pp. $119-149$.

[2] Aouf M. K., Dziok J., Sokół J. On a subclass of strongly starlike functions. Appl. Math. Letters., 2011, vol. 24, pp. 27-32.

DOI: https://doi.org/10.1016/j.aml.2010.08.004.

[3] Babalola K. O. On $\mathrm{H}_{3}(1)$ Hankel determinant for some classes of univalent functions. Inequality Theory \& Applications, vol. 6, 2010, pp. 1-7.

DOI: https://doi.org/10.1I55/2008/153280.

[4] Dziok J., Raina R. K., Sokół J. On a class of starlike functions related to a shell-like curve connected with Fibonacci numbers. Math. Comput. Modelling., 2013, vol. 57, pp. 1203-1211.

DOI: https://doi.org/10.1016/j.mcm.2012.10.023. 
[5] Dziok J., Raina R. K., Sokół J. Certain results for a class of convex functions related to a shell-like curve connected with Fibonacci numbers. Comput. Math. Appl., 2011, vol. 61, no. 9, pp. 2605-2613.

DOI: https://doi.org/10.1016/j. camwa.2011.03.006.

[6] Fekete M., Szegö G. Eine bemerkung uber ungerade schlichte function. J.London Math.Soc., 1933, vol. 8, pp. 85-89.

DOI: https://doi.org/10.1112/jlms/s1-8.2.85.

[7] Janteng A., Halim S. A., Darus M. Coefficient inequality for a function whose derivative has a positive real part. J. Inequal. Pure Appl. Math., 2006, vol. 7, no. 2, Article 50.

[8] Janteng A., Halim S. A., Darus M. Hankel determinant for starlike and convex functions. Int. J. Math. Anal., 2007, vol. 1, no 13, pp. 619-625.

[9] Keogh F. R., Merkes E. P. A coefficient inequality for certain classes of analytic functions. Proc. Amer. Math. Soc., 1969, vol. 20, pp. 8-12.

DOI: https://doi.org/10.2307/2035949.

[10] Krushkal S. L. Proof of the Zalcman conjecture for initial coefficients. Georgian Math. J., 2010, vol. 17, pp. 663-681.

DOI: https://doi.org/10.1515/GMJ .2010.043.

[11] Ma W. Generalized Zalcman conjecture for starlike and typically real functions. J. Math. Anal. Appl., 1999, vol. 234 no. 1, pp. 328-339.

DOI: https://doi.org/10.1006/jmaa.1999.6378.

[12] Pommerenke Ch. Univalent functions. Vandenhoeck and Rupercht, Gotingen, 1975.

[13] Raina R. K, Sokół J. on coefficient estimates for a certain class of starlike functions. Hacet. J. Math. Stat., 2015, vol. 44, no 6, pp. 1427-1433.

DOI: https://doi.org/10.15672/HJMS . 2015449676.

[14] Ravichandran V., Verma S. Generalized Zalcman conjecture for some classes of analytic functions. J. Math. Anal. Appl., 2017, vol. 450, no. 1, pp. $592-605$.

DOI: https://doi.org/10.1016/j.jmaa.2017.01.053.

[15] Sharma R. B., Haripriya M. On a class of $\alpha$-convex functions subordinate to a shell shaped region. The J. Anal., 2017, vol. 25, no 1, pp. 99-105.

DOI: https://doi.org/10.1007/s41478-017-0031-z.

[16] Srivastava H. M., Mishra A. K., Das M. K. The Fekete-Szegö problem for subclass of close-to-convex functions. Complex Var. Theory Appl., 2001, vol. 44, no. 2, pp. $145-163$.

DOI: https://doi.org/10.1080/17476930108815351. 
[17] Srivastava H. M., Lei S., Arif M., Hussain S., Khan H. An investigation of the third Hankel determinant problem for certain subfamilies of univalent functions involving the exponential function. Symmetry., 2019, vol. 11, no. 5 , pp. 598.

DOI: https://doi.org/10.3390/sym11050598.

[18] Vamshee Krishna D., Ram Reddy T. Coefficient inequalities for certain subclasses of analytic functions associated with Hankel determinant. Indian J. Pure Appl. Math., 2015, vol. 46, no. 1, pp. 91-106.

DOI: https://doi.org/10.1007/s13226-015-0111-1.

Received September 0\%, 2019.

In revised form, February 28, 2020.

Accepted March 03, 2020.

Published online March 27, 2020.

\section{Suman Kumar}

Department of Mathematics, TSMS, Chigurumamidi, Karimnagar, Telangana-505481, India

E-mail: suman.vodnala@gmail.com

R. B. Sharma

Department of Mathematics, Kakatiya University, Warangal, Telangana-506009, India

E-mail: rbsharma005@gmail.com 